\title{
Fast approximate solution of Bloch equation for simulation of RF artifacts in Magnetic Resonance Imaging
}

\author{
S. Balac, L. Chupin \\ Institut Camille Jordan (UMR CNRS 5208) \\ INSA de Lyon, 69621 Villeurbanne, France
}

\section{SUMMARY}

The technique used to spot information in Magnetic Resonance Imaging (MRI) uses electromagnetic fields. Even minor perturbations of these magnetic fields can disturb the imaging process and may render clinical images inaccurate or useless. Modelling and numerical simulation of the effects of static field inhomogeneities are now well established. Less attention has been paid to mathematical modeling of the effects of radio-frequency (RF) field inhomogeneities in the imaging process. When considering RF field inhomogeneities, the major difficulty is that the mathematical expression of the magnetisation vector is not anymore explicitly known contrarily to the unperturbed case. Indeed, the Bloch equation becomes an ordinary differential equation with non constant coefficients that cannot be solved analytically. The use of standard numerical schemes for ordinary differential equations to compute the magnetisation vector appears to be costly and not well suited for MRI image simulation. In this paper, we present an original method for solving the Bloch equation based on a truncated series expansion of the solution. The computational cost of the method reduces to the computation of the eigen-elements of a block tridiagonal matrix of a very small size.

KEY WORDS: Bloch equation, magnetic resonance imaging, Fourier series expansion, Floquet theory

\section{Introduction}

Magnetic Resonance Imaging (MRI) is a method that generates exquisite images of the soft tissue anatomy of the human body. The principle of MRI is to record the variations of the nuclear magnetisation of the biological tissues by using different kinds of magnetic fields, see [1],[2]. A static magnetic field $\mathbf{B}_{\mathbf{0}}$ is used to generate a macroscopic nuclear magnetisation $\mathbf{M}$ in the body to be imaged; typically $\mathbf{B}_{\mathbf{0}}$ has a strength around 1 Tesla. This equilibrium magnetisation is aligned with $\mathbf{B}_{\mathbf{0}}$. To shift the magnetisation vector $\mathbf{M}$ from its equilibrium position, a radio-frequency magnetic field ( $\mathrm{RF}$ field) $\mathbf{B}_{\mathbf{1}}$ is applied at a very characteristic pulsation determined by the Larmor relation

$$
\omega_{0}=\gamma\left\|\mathbf{B}_{0}\right\|
$$

*Correspondence to: S. Balac or L. Chupin, Institut Camille Jordan, Bât. L. de Vinci, 21 av. Capelle, INSA de Lyon, 69621 Villeurbanne cedex, France. 
where $\gamma$ is a constant called the gyro-magnetic ratio ( $\gamma=42.58 \mathrm{Mhz}$ by Tesla for proton). In MRI this phenomenon is known as the resonance process. The position of the magnetisation vector at the end of the resonance process is determined by the duration of the RF field $\mathbf{B}_{\mathbf{1}}$. Typically this duration is chosen so that the angle between the initial position and the resulting one is $\pi / 2$ or $\pi$. When the RF field $\mathbf{B}_{\mathbf{1}}$ is stopped, the magnetisation tends to return to its equilibrium position in a process called the relaxation. During the relaxation process the magnetisation creates an induced electric signal in an antenna set in a plane perpendicular to $\mathbf{B}_{\mathbf{0}}$. This signal is acquired for subsequent processing and gives rise to the image. Moreover, magnetic field gradients (static magnetic field aligned with $\mathbf{B}_{\mathbf{0}}$ with a linear varying intensity in one fixed direction) are applied during the imaging process to set up a spatial correspondence between position in the body and position in the image through a frequency encoding of the MRI signal.

Any perturbation of the magnetic fields involved in MRI can disturb the imaging process. The result is a local deformation of the image (called an artifact) that may render the image inaccurate and useless for medical diagnostics. Sources of perturbation of the magnetic fields are various and can be classified in two groups: the one connected to the static magnetic field and the one connected to the RF field. Moreover one can distinguish between defects that are properties of the MRI device (e.g. non uniformity of the magnetic fields over the whole imaging area) and perturbations of the magnetic fields due to the patient himself. Since they are fixed properties of the MRI device, the first ones can be handled with efficiency, either by the use of additional hardware components [3][4] or by taking into account their effect in the reconstruction algorithm. It is much more difficult to deal with the second ones as they are not identical from one experiment to the other. Common causes of such magnetic field perturbations are changes of magnetic properties in the sample due to metallic implanted objects, such as dental prostheses, hip prostheses, vascular clips, internal orthopaedic devices, etc. Also, metallic surgical instruments used in interventional MRI are responsible for such magnetic field perturbations.

Artifacts produced by the magnetic susceptibility of metallic implants have been widely studied in the literature using an experimental approach, see [5] for a review, as well as using mathematical modelling and numerical simulation, see [6] [7]. Less attention has been paid to MRI artifacts caused by eddy currents in conducting metallic implants. Eddy currents may originate either from the RF field $\mathbf{B}_{\mathbf{1}}$ or from the field gradients. However it has been found, see [8], that artifacts due to eddy currents from the magnetic field gradients are not significant. Past studies on artifacts from RF field induced by eddy currents have been mostly qualitative and experimental, see [9] [10] [11]. In this paper we focus on the modelling of RF artifacts due to eddy currents in metallic conductive object. An important property of RF fields induced by eddy currents in a metallic conductive object is that it has the same frequency as the main RF field $\mathbf{B}_{\mathbf{1}}$. Moreover the strength of the RF field $\mathbf{B}_{\mathbf{1}}^{\prime}$ is of the same order than the main RF field $\mathbf{B}_{\mathbf{1}}$ and decreases in space from the boundary of the object as the power 3 of the distance.

In order to perform numerical simulation of RF artifact it is necessary to solve two distinct problems. The first one is to compute the RF field disturbances due to the metallic implant; for simple test objects (such as cylinders, spheres or ellipsoids) analytical expressions for the RF field perturbation are known, see [11] [10]. In general a precise calculation of the RF field perturbation involves a boundary value problem with partial differential equation derived from Maxwell's equations and requires the use of PDE approximation schemes. Among the classical methods are the finite element method, the finite difference method and the boundary element 
method. For a comprehensive treatment of these methods in electromagnetism we refer to [12] and [13]. The second problem, once the RF perturbations are known, is to determine how the evolution of the magnetisation is disturbed during the resonance and relaxation processes and give rise to the artifacts. Time dependence of the macroscopic nuclear magnetisation $\mathbf{M}$ under the influence of a magnetic flux density $\mathbf{B}$ is modelled by the following differential equation proposed in 1946 by F. Bloch, see [14] [1],

$$
\frac{\mathrm{d} \mathbf{M}}{\mathrm{d} t}=\gamma(\mathbf{M} \wedge \mathbf{B})-\frac{M_{x} \mathbf{x}+M_{y} \mathbf{y}}{T_{2}}-\frac{M_{z}-\eta_{0}}{T_{1}} \mathbf{z},
$$

where $\left(M_{x}, M_{y}, M_{z}\right)$ denotes the components of the magnetisation $\mathbf{M}$ in the laboratory frame $(\mathbf{x}, \mathbf{y}, \mathbf{z}), T_{1}$ and $T_{2}$ known respectively as the spin-lattice and spin-spin relaxation times measure the interaction of the nuclei with their surrounding molecular environment and those between close nuclei and $\eta_{0}$ is the strength of the equilibrium magnetisation. This equation is valid during the resonance process as well as during the relaxation process, provided that the correct form for the total magnetic flux density $\mathbf{B}$ is taken $\left(\mathbf{B}=\mathbf{B}_{\mathbf{0}}+\mathbf{B}_{\mathbf{1}}\right.$ during the resonance process and $\mathbf{B}=\mathbf{B}_{\mathbf{0}}$ during the relaxation process when the $\mathrm{RF}$ field is stopped).

The basic idea for numerical simulation of MRI images is to solve repeatedly the Bloch equation for each volume element (voxel) in the sample. Actually for each voxel the Bloch equation has to be considered twice. The Bloch equation has to be solved a first time during the resonance process to get the position of the magnetisation vector at the end of the resonance. It is during this stage that we have to take into account the effects of RF field perturbations. The position of the magnetisation vector at the end of the resonance process is the initial position of the magnetisation vector for the relaxation process. Then the Bloch equation has to be solved a second time during the relaxation process. As the RF field $\mathbf{B}_{\mathbf{1}}$ is stopped during the relaxation, there is no RF field disturbances to be taken into account for this stage.

In the ideal case where no disturbance of the RF field $\mathbf{B}_{\mathbf{1}}$ occurs, the Bloch equation can be solved analytically, see [15]. In a frame in rotation around $\mathbf{z}$ at Larmor frequency, the Bloch equation reduces to a system of linear differential equations with constant coefficients. The position of the magnetisation at the end of the resonance process can therefore be determined in a straightforward way without expensive computation. Under RF field perturbations such an approach is not anymore possible: the Bloch equation can not be reduced to a system of linear equations with constant coefficients in an appropriate frame. It means that for each voxel the Bloch equation needs to be solved using a numerical discretisation scheme and it is then very costly to obtain a good resolution image. Typically, the Bloch equation can be solved using Runge-Kutta methods. A drawback of such numerical methods to approximate the solution of a differential equation is that to compute the solution at a given time $T$ (for our concern, the end of the resonance process) the solution has to be computed over the whole interval $[0, T]$ at different discretisation times and the accuracy of the solution at time $T$ strongly depend on the size of the discretisation step. Moreover an important consideration in using numerical methods to approximate the solution of a differential equation is the stability of the method.

In this paper we present an original method to compute solution $\mathbf{M}$ to the bloch equation under RF field inhomogeneities at a given time $T$ directly, without any discretisation of the time interval $[0, T]$. The method is based on a series expansion of the solution. Through this transform, the Bloch equation is changed of into an infinite system of linear differential equations with constant coefficients, the unknowns being the coefficients of the series expansion of $\mathbf{M}$. The efficiency of the method stands in the fact that the infinite system can be truncated 
and a very accurate solution can be obtained with only a few terms in the series expansion. The cost of the method is the computation of eigenvalues and eigenvectors of a block tridiagonal matrix of small size (for magnetic field values commonly used in MRI, this matrix is of size $9 \times 9)$.

The paper is organised as follows. The next section is devoted to a presentation of the Bloch equation in the framework of MRI and to the way it can be solved. In section 3, the mathematical features of our method are presented and convergence estimates are derived. In section 4 we present the algorithm to solve the Bloch equation and compare its efficiency to other numerical schemes.

\section{Bloch equation under RF perturbations}

\subsection{Bloch equation in the rotating frame}

We are concerned with the Bloch equation in the situation where a perturbation of the RF magnetic field is superimposed to the usual magnetic fields used in the MRI experiment. During the resonance process, the total magnetic field in a voxel located at a position $\mathbf{r}$ is given in the laboratory frame $(\mathbf{x}, \mathbf{y}, \mathbf{z})$ by

$$
\mathbf{B}(\mathbf{r}, t)=\mathbf{B}_{\mathbf{0}}+\mathbf{B}_{\mathbf{1}}(t)+\mathbf{B}_{\mathbf{1}}^{\prime}(\mathbf{r}, t),
$$

where $\mathbf{B}_{\mathbf{0}}=B_{0} \mathbf{z}$ is a constant field, $\mathbf{B}_{\mathbf{1}}(t)=B_{1} \cos \left(\omega_{0} t\right) \mathbf{x}-B_{1} \sin \left(\omega_{0} t\right) \mathbf{y}$ is the RF magnetic field whereas the perturbation $\mathrm{RF}$ field is given by

$$
\begin{aligned}
\mathbf{B}_{\mathbf{1}}^{\prime}(\mathbf{r}, t) & =\left(u_{1}(\mathbf{r}) \cos \left(\omega_{0} t\right)+v_{1}(\mathbf{r}) \sin \left(\omega_{0} t\right)\right) \mathbf{x}+\left(u_{2}(\mathbf{r}) \cos \left(\omega_{0} t\right)+v_{2}(\mathbf{r}) \sin \left(\omega_{0} t\right)\right) \mathbf{y} \\
& +\left(u_{3}(\mathbf{r}) \cos \left(\omega_{0} t\right)+v_{3}(\mathbf{r}) \sin \left(\omega_{0} t\right)\right) \mathbf{z} .
\end{aligned}
$$

Typically, this latter expression can represent the RF field generated by eddy currents in a metallic conducting object lying in the MRI experiment area (metallic implant, catheter, ....). The eddy current is a swirling current set up in the conductor in response to the changing RF field $\mathbf{B}_{\mathbf{1}}$. It creates, according to Lenz's law, a magnetic RF field opposing the change. The magnetic RF field induced by eddy currents has a frequency identical to the one of the imposed RF field $\mathbf{B}_{\mathbf{1}}$ but the greater the electrical conductivity of the conductor, the stronger the intensity of the induced field.

Inhomogeneities of the static magnetic field as well as magnetic field gradients can be included in the modelling by taking the following form for $\mathbf{B}_{\mathbf{0}}: B_{0, x}^{\prime}(\mathbf{r}) \mathbf{x}+B_{0, y}^{\prime}(\mathbf{r}) \mathbf{y}+\left(B_{0, z}^{\prime}(\mathbf{r})+\right.$ $\left.B_{0}\right) \mathbf{z}$. For convenience they won't be considered in the following. Component-wise, the Bloch equation (2) reads

$$
\left\{\begin{aligned}
\frac{\mathrm{d}}{\mathrm{d} t} M_{x}(\mathbf{r}, t) & =\gamma\left(M_{y}(\mathbf{r}, t) B_{z}(\mathbf{r}, t)-M_{z}(\mathbf{r}, t) B_{y}(\mathbf{r}, t)\right)-\frac{M_{x}(\mathbf{r}, t)}{T_{2}(\mathbf{r})} \\
\frac{\mathrm{d}}{\mathrm{d} t} M_{y}(\mathbf{r}, t) & =\gamma\left(M_{z}(\mathbf{r}, t) B_{x}(\mathbf{r}, t)-M_{x}(\mathbf{r}, t) B_{z}(\mathbf{r}, t)\right)-\frac{M_{y}(\mathbf{r}, t)}{T_{2}(\mathbf{r})} \\
\frac{\mathrm{d}}{\mathrm{d} t} M_{z}(\mathbf{r}, t) & =\gamma\left(M_{x}(\mathbf{r}, t) B_{y}(\mathbf{r}, t)-M_{y}(\mathbf{r}, t) B_{x}(\mathbf{r}, t)\right)-\frac{M_{z}(\mathbf{r}, t)-\eta_{0}(\mathbf{r})}{T_{1}(\mathbf{r})}
\end{aligned}\right.
$$

where the components $\left(B_{x}, B_{y}, B_{z}\right)$ of $\mathbf{B}$ are given by $B_{x}(\mathbf{r}, t)=\left(B_{1}+u_{1}(\mathbf{r})\right) \cos \left(\omega_{0} t\right)+$ $v_{1}(\mathbf{r}) \sin \left(\omega_{0} t\right), B_{y}(\mathbf{r}, t)=\left(-B_{1}+v_{2}(\mathbf{r})\right) \sin \left(\omega_{0} t\right)+u_{2}(\mathbf{r}) \cos \left(\omega_{0} t\right)$, and $B_{z}(\mathbf{r}, t)=B_{0}+$ 
$u_{3}(\mathbf{r}) \cos \left(\omega_{0} t\right)+v_{3}(\mathbf{r}) \sin \left(\omega_{0} t\right)$. For convenience, from now on we drop the spatial dependence $\mathbf{r}$ and denote $\mathbf{M}(t)$ the magnetisation vector at time $t$. As well, we set $\tau_{1}=T_{1}^{-1}$ and $\tau_{2}=T_{2}^{-1}$. For biological tissues, the relaxation time $T_{1}$ may vary from 100 millisecond to 1 second (e.g. $750 \mathrm{~ms}$ for muscle and $250 \mathrm{~ms}$ for fat at 1 Tesla) whereas the relaxation time $T_{2}$ may vary from 50 to 100 millisecond (e.g. $50 \mathrm{~ms}$ for muscle and $80 \mathrm{~ms}$ for fat at 1 Tesla). In the situation considered here, the RF magnetic field perturbation $\mathbf{B}_{\mathbf{1}}^{\prime}$ is assumed to have a magnitude in the same range as the original $\mathrm{RF}$ magnetic field $\mathbf{B}_{\mathbf{1}}$, namely $10^{-4}$ to $10^{-3}$ Tesla. As a consequence, since $\mathbf{B}_{\mathbf{0}}$ is around 1 Tesla, the following approximation will be done: $B_{z}(\mathbf{r}, t) \approx B_{0}$.

In matrix form, the Bloch equation reads

$$
\frac{\mathrm{d}}{\mathrm{d} t} \mathbf{M}(t)=A(t) \mathbf{M}(t)+\mathbf{b}
$$

where

$$
A(t)=\left(\begin{array}{ccc}
-\tau_{2} & \gamma B_{0} & -\gamma B_{y}(t) \\
-\gamma B_{0} & -\tau_{2} & \gamma B_{x}(t) \\
\gamma B_{y}(t) & -\gamma B_{x}(t) & -\tau_{1}
\end{array}\right) \quad \text { and } \quad \mathbf{b}=\left(\begin{array}{c}
0 \\
0 \\
\tau_{1} \eta_{0}
\end{array}\right)
$$

Let us observe that the matrix $A$ is not suited for the resolution of the differential system by numerical method. Indeed, its coefficients $(1,2)$ and $(2,1)$ are of magnitude $10^{6}$ whereas the others are of magnitude 1 . By a change of unknown, it is possible to balance the coefficients of the matrix of the differential system. Namely, if we set $\mathbf{M}(t)=R(t) \mathbf{m}(t)$ with $R(t)$ the following matrix rotation around $\mathbf{z}$

$$
R(t)=\left(\begin{array}{ccc}
\cos \left(\omega_{0} t\right) & \sin \left(\omega_{0} t\right) & 0 \\
-\sin \left(\omega_{0} t\right) & \cos \left(\omega_{0} t\right) & 0 \\
0 & 0 & 1
\end{array}\right)
$$

then $\mathbf{m}=\left(m_{1}, m_{2}, m_{3}\right)^{\mathrm{T}}$ satisfies the generalised differential system

$$
R(t) \frac{\mathrm{d}}{\mathrm{d} t} \mathbf{m}(t)=\left(A(t) R(t)-\frac{\mathrm{d}}{\mathrm{d} t} R(t)\right) \mathbf{m}(t)+\mathbf{b} .
$$

Since $R^{-1} \mathbf{b}=\mathbf{b}$, this differential system reads

$$
\frac{\mathrm{d}}{\mathrm{d} t} \mathbf{m}(t)=\tilde{A}(t) \mathbf{m}(t)+\mathbf{b}
$$

where

$$
\tilde{A}(t)=P^{-1}\left(A P-\frac{\mathrm{d}}{\mathrm{d} t} P\right)=\left(\begin{array}{ccc}
-\tau_{2} & 0 & -\omega_{a}(t) \\
0 & -\tau_{2} & \omega_{b}(t) \\
\omega_{a}(t) & -\omega_{b}(t) & -\tau_{1}
\end{array}\right)
$$

with

$$
\begin{aligned}
\omega_{a}(t) & =\gamma B_{y}(t) \cos \left(\omega_{0} t\right)+\gamma B_{x}(t) \sin \left(\omega_{0} t\right), \\
\omega_{b}(t) & =\gamma B_{x}(t) \cos \left(\omega_{0} t\right)-\gamma B_{y}(t) \sin \left(\omega_{0} t\right) .
\end{aligned}
$$

As $w_{a}$ and $w_{b}$ are regular functions of time, it's follows from Cauchy-Lipschitz theorem (see [16]) that the first order linear differential system (9) has a unique solution under the initial condition $\mathbf{m}(0)=\mathbf{M}_{\mathbf{0}}$. 


\subsection{Solving Bloch equation without RF perturbation}

Let us observe that without RF perturbation $\left(u_{1}=v_{1}=u_{2}=v_{2}=0\right)$, the matrix $\tilde{A}(t)$ reduces to the following constant matrix

$$
\tilde{A}=\left(\begin{array}{ccc}
-\tau_{2} & 0 & 0 \\
0 & -\tau_{2} & \omega_{1} \\
0 & -\omega_{1} & -\tau_{1}
\end{array}\right)
$$

where $\omega_{1}=\gamma B_{1}$ and the differential system (9) can be solved analytically using standard results from differential equations theory [16][17]. The matrix $\tilde{A}$ has three distinct eigenvalues : $\lambda_{0}=-\tau_{2}$ and

$$
\lambda_{ \pm}=-\frac{1}{2}\left(\tau_{1}+\tau_{2}\right) \pm \frac{1}{2} \mathrm{i} \sqrt{4 \omega_{1}^{2}-\left(\tau_{1}-\tau_{2}\right)^{2}} .
$$

As the quantity $4 \omega_{1}^{2}-\left(\tau_{1}-\tau_{2}\right)^{2}$ is positive (this property is connected to the values of the physical quantities involved) the eigenvalues $\lambda_{+}$and $\lambda_{-}$are complex conjugated numbers. The eigenvectors associated to the three eigenvalues $\lambda_{0}, \lambda_{+}$and $\lambda_{-}$are respectively

$$
\mathbf{v}_{\mathbf{0}}=\left(\begin{array}{l}
1 \\
0 \\
0
\end{array}\right), \quad \mathbf{v}_{+}=\left(\begin{array}{c}
0 \\
\tau_{1}+\lambda_{+} \\
-\omega_{1}
\end{array}\right) \quad \text { and } \quad \mathbf{v}_{-}=\left(\begin{array}{c}
0 \\
\tau_{1}+\lambda_{-} \\
-\omega_{1}
\end{array}\right) .
$$

The solution to the homogeneous differential system associated to (9) is then

$$
\mathbf{m}_{\mathbf{h}}(t)=C_{0} \exp \left(\lambda_{0} t\right) \mathbf{v}_{\mathbf{0}}+C_{+} \exp \left(\lambda_{+} t\right) \mathbf{v}_{+}+C_{-} \exp \left(\lambda_{-} t\right) \mathbf{v}_{-} \quad \text { with }\left(C_{0}, C_{+}, C_{-}\right) \in \mathbb{C}^{3}
$$

whereas a particular solution is found to be

$$
\mathbf{m}_{\mathbf{p}}(t)=\left(\begin{array}{c}
0 \\
-\frac{\omega_{1} \tau_{1}}{\tau_{1} \tau_{2}+\omega_{1}^{2}} \eta_{0} \\
-\frac{\tau_{1} \tau_{2}}{\tau_{1} \tau_{2}+\omega_{1}^{2}} \eta_{0}
\end{array}\right)
$$

The solution to the differential system $(9)$ is then $\mathbf{m}(t)=\mathbf{m}_{\mathbf{h}}(t)+\mathbf{m}_{\mathbf{p}}(t)$ where the three constants $C_{0}, C_{+}$and $C_{-}$are determined by the initial value $\mathbf{M}_{\mathbf{0}}$ of the magnetisation vector $\mathbf{M}$.

A look at the values of the different physical quantities involved shows that $\tau_{1}$ and $\tau_{2}$ can be neglected compared to $\omega_{1}$ in (12) and the solution $\mathbf{m}$ to the differential system (9) under the initial condition $\mathbf{M}_{\mathbf{0}}=\eta_{0} \mathbf{z}$ is therefore:

$$
\mathbf{m}(t)=\left(\begin{array}{c}
0 \\
\eta_{0} \sin \omega_{1} t \\
\eta_{0} \cos \omega_{1} t
\end{array}\right)
$$

Relation (15) has the following interpretation: in the rotating frame $\left(\mathbf{e}_{\mathbf{1}}, \mathbf{e}_{\mathbf{2}}, \mathbf{e}_{\mathbf{3}}\right)$ image of the laboratory frame $(\mathbf{x}, \mathbf{y}, \mathbf{z})$ by the rotation $R(t)$, the magnetisation vector starts to flip out of the $\mathbf{z}$ axis from its equilibrium position $\mathbf{M}_{\mathbf{0}}=\eta_{0} \mathbf{z}$ under the influence of the RF field $\mathbf{B}_{\mathbf{1}}$. The flip angle between the magnetisation vector and the $\mathbf{z}$ axis is a function of time given by $\theta(t)=\omega_{1} t$. The magnetisation vector rotates around the $\mathbf{e}_{\mathbf{1}}$ axis with the angular frequency $\omega_{1}$. 
If the $\mathrm{RF}$ field $\mathbf{B}_{\mathbf{1}}$ is applied for a duration $T_{R F}$, then the magnetisation vector $\mathbf{M}$ is tilt over an angle

$$
\Theta=\theta\left(T_{R F}\right)=\omega_{1} T_{R F} .
$$

The two flip angles commonly used in MRI are $\Theta=\pi / 2$ and $\Theta=\pi$. According to (16), they are obtained by applying the RF flux density $\mathbf{B}_{\mathbf{1}}$ for a duration $T_{R F}=\frac{\pi}{2 \omega_{1}}$ and $T_{R F}=\frac{\pi}{\omega_{1}}$ respectively. In the laboratory frame the components of the magnetisation vector $\mathbf{M}(t)=\stackrel{\omega_{1}}{\mathcal{R}}(t) \mathbf{m}(t)$ are given by :

$$
\mathbf{M}(t)=\left(\begin{array}{ccc}
\eta_{0} & \sin \left(\omega_{1} t\right) & \sin \left(\omega_{0} t\right) \\
\eta_{0} & \sin \left(\omega_{1} t\right) & \cos \left(\omega_{0} t\right) \\
& \eta_{0} & \cos \left(\omega_{1} t\right)
\end{array}\right) .
$$

Let us mention that during the relaxation process, the RF field $\mathbf{B}_{\mathbf{1}}$ is stopped (and so RF field perturbations cancel). The magnetisation vector $\mathbf{M}$ still evolves according to the Bloch equation (5) but now the matrix $A(t)$ is reduced to the constant matrix

$$
A=\left(\begin{array}{ccc}
-\tau_{2} & \gamma B_{0} & 0 \\
-\gamma B_{0} & -\tau_{2} & 0 \\
0 & 0 & -\tau_{1}
\end{array}\right)
$$

Thus, the evolution of the magnetisation vector during the relaxation process is always explicitly known and given in the laboratory frame by

$$
\mathbf{M}(t)=\left(\begin{array}{c}
\mathrm{e}^{-\tau_{2} t}\left(C_{1} \cos \left(\omega_{0} t\right)+C_{2} \sin \left(\omega_{0} t\right)\right) \\
\mathrm{e}^{-\tau_{2} t}\left(C_{2} \cos \left(\omega_{0} t\right)-C_{1} \sin \left(\omega_{0} t\right)\right) \\
\eta_{0}-C_{3} \mathrm{e}^{-\tau_{1} t}
\end{array}\right)
$$

where the three constants $C_{1}, C_{2}$ and $C_{3}$ are determined by the value of the magnetisation vector at the beginning of the relaxation.

\subsection{Overview of the method for solving Bloch equation}

Under RF perturbation, the matrix $\tilde{A}$ is not constant and the differential system (9) can not be solved analytically. However, the matrix $\tilde{A}$ is continuous and has periodic coefficients of period $T_{0}=2 \pi / \omega_{0}$. According to Floquet theory (see [17]), the fundamental solution $X(t)$ for the differential system (9) has the following expression:

$$
X(t)=Q(t) \mathrm{e}^{t F}
$$

where $Q$ is a matrix with continuous and periodic coefficients of period $T_{0}$ and $F$ is a constant matrix. Floquet theory gives no practical information about a way to compute the matrices $Q$ and $F$ and actually there exists no general method to compute them. There are two ways of exploiting the Floquet structure of $X(t)$, see [18][19][20]. The first one consists in performing a Fourier expansion of the fundamental solution, leading to an infinite system of linear differential equations with constant coefficients. When the constant coefficients have adequate properties, resolution of a truncated system furnishes an approximate solution. The second approach is of perturbative nature and deals with the Floquet form by expanding the two matrices $Q$ and $F$ as $Q(t)=\sum_{n=1}^{+\infty} Q_{n}(t)$ and $F=\sum_{n=1}^{+\infty} F_{n}$ where every term $F_{n}$ is chosen in order to ensure the matrix $Q_{n}(t)$ is periodic and in turn $Q_{n}(t)$ is fixed so as to guarantee the Floquet structure at any order of truncation. 
Our approach does not directly use the Floquet form of the fundamental solution but exploits some of the ideas of the two approaches presented above in the following way. First, let us observe that the matrix $\tilde{A}(t)$ admits the following Fourier decomposition: $\tilde{A}(t)=\sum_{k=-1}^{1} A_{2 k} \mathrm{e}^{2 \mathrm{i} k \omega_{0} t}=A_{-2} \mathrm{e}^{-2 \mathrm{i} \omega_{0} t}+A_{0}+A_{2} \mathrm{e}^{2 \mathrm{i} \omega_{0} t}$ where

$$
A_{0}=\left(\begin{array}{ccc}
-\tau_{2} & 0 & -w_{a}^{(0)} \\
0 & -\tau_{2} & w_{b}^{(0)} \\
w_{a}^{(0)} & -w_{b}^{(0)} & -\tau_{1}
\end{array}\right) \quad \text { and } \quad A_{2}=\left(\begin{array}{ccc}
0 & 0 & -\omega_{a}^{(2)} \\
0 & 0 & \omega_{b}^{(2)} \\
\omega_{a}^{(2)} & -\omega_{b}^{(2)} & 0
\end{array}\right)
$$

with

$$
\begin{array}{ll}
\omega_{a}^{(0)}=\frac{1}{2} \gamma\left(u_{2}+v_{1}\right), & \omega_{a}^{(2)}=-\frac{1}{4} \gamma\left(v_{1}-u_{2}+\mathrm{i}\left(u_{1}+v_{2}\right)\right), \\
\omega_{b}^{(0)}=\frac{1}{2} \gamma\left(2 B_{1}+u_{1}-v_{2}\right), & \omega_{b}^{(2)}=\frac{1}{4} \gamma\left(u_{1}+v_{2}+\mathrm{i}\left(u_{2}-v_{1}\right)\right),
\end{array}
$$

and $A_{-2}=\overline{A_{2}}$ is the conjugate matrix of $A_{2}$. As the solution $\mathbf{m}(t)$ of $(9)$ is not periodic it is not possible to compute it through its Fourier series expansion. Thus, we look for a formal solution of the following type:

$$
\mathbf{m}(t)=\sum_{k \in \mathbb{Z}} \mathbf{m}_{k}(t) e^{2 \mathrm{i} k \omega_{0} t}
$$

which looks like a Fourier series expansion but with non constant coefficients $\mathbf{m}_{k}(t)$. This decomposition is not unique since no condition is imposed on the functions $\mathbf{m}_{k}$. If we use the expansion (22) in the differential system (9) we obtain $\sum_{k \in \mathbb{Z}} \mathbf{r}_{k}(t) e^{2 \mathrm{i} k \omega_{0} t}=0$ where

$$
\mathbf{r}_{k}(t)=\frac{\mathrm{d}}{\mathrm{d} t} \mathbf{m}_{k}(t)-\sum_{j=-1}^{1} A_{2 j} \mathbf{m}_{k-j}(t)+2 \mathrm{i} k \omega_{0} \mathbf{m}_{k}(t)-\delta_{k} \mathbf{b}
$$

with the sequence $\left(\delta_{k}\right)_{k}$ given by $\delta_{0}=1$ and $\delta_{k}=0$ for $k \in \mathbb{Z}^{*}$. Contrary to a standard Fourier expansion, it can not be deduced that $\mathbf{r}_{k}(t)=0$ for all $k \in \mathbb{Z}$. Nevertheless, if we can solve all equations $\mathbf{r}_{k}(t)=0$ under appropriate initial conditions so that the series $\sum_{k \in \mathbb{Z}} \mathbf{m}_{k}(t) e^{2 \mathrm{i} k \omega_{0} t}$ converges then the series expansion $\mathbf{m}(t)$ as given by (22) will be solution of the differential system (9). If $\sum_{k \in \mathbb{Z}} \mathbf{m}_{k}(0)=\mathbf{M}_{\mathbf{0}}$ then $\mathbf{m}(t)$ will be the solution to the differential system (9) under the initial condition $\mathbf{m}(0)=\mathbf{M}_{\mathbf{0}}$.

The next section is devoted to the study of the following infinite sequence of differential equations:

$$
\forall k \in \mathbb{Z} \quad\left\{\begin{array}{l}
\frac{\mathrm{d}}{\mathrm{d} t} \mathbf{m}_{k}(t)=\sum_{j=-1}^{1} A_{2 j} \mathbf{m}_{k-j}(t)-2 \mathrm{i} k \omega_{0} \mathbf{m}_{k}(t)+\delta_{k} \mathbf{b} \\
\mathbf{m}_{k}(0)=\delta_{k} \mathbf{M}_{\mathbf{0}}
\end{array}\right.
$$

We will show that (23) admits a unique solution and that for a given time $t$ the sequence $\left(\mathbf{m}_{k}(t)\right)_{k \in \mathbb{Z}}$ converges very quickly towards zero when $k$ tends to $\pm \infty$. This analysis of the behaviour of the solution to the sequence of differential equations (23) will justify the fact that an approximation of the solution to the Bloch equation can be computed by solving a truncated finite differential system deduced from (23) by taking $k \in\{-N, \ldots, N\}$. 
3. Analysis of the behaviour of the sequence $\left(\mathbf{m}_{k}\right)_{k \in \mathbb{Z}}$

\subsection{Mathematical framework}

In order to carry on the mathematical analysis of the sequence of differential equations (23), we first have to introduce a suitable mathematical framework. A given sequence $\left(\mathbf{u}_{k}\right)_{k \in \mathbb{Z}}$ of vectors in $\mathbb{C}^{3}$ can be seen as an infinite vector, denoted by $\mathcal{U}$ in the sequel, with the $k$ th component given by $\mathcal{U}_{k}=\mathbf{u}_{k} \in \mathbb{C}^{3}$. We denote by $\ell^{2}(\mathbb{Z})$ the space of square summable series

$$
\ell^{2}(\mathbb{Z})=\left\{\mathcal{U} \in\left(\mathbb{C}^{3}\right)^{\mathbb{Z}} ; \sum_{k \in \mathbb{Z}}\left|\mathbf{u}_{k}\right|^{2}<+\infty\right\}
$$

and by $\ell^{1}(\mathbb{Z})$ the corresponding space of absolute convergent series where $\left|\mathbf{u}_{k}\right|$ denotes the euclidean norm of $\mathbf{u}_{k}$ in $\mathbb{C}^{3}$. For any positive real number $p$ we defined the following space of weighted square summable series:

$$
\ell_{p}^{2}(\mathbb{Z})=\left\{\mathcal{U} \in \ell^{2}(\mathbb{Z}) ; \sum_{k \in \mathbb{Z}}\left(k^{2 p}+1\right)\left|\mathbf{u}_{k}\right|^{2}<+\infty\right\} .
$$

This space is equipped with the scalar product $<,>_{\ell_{p}^{2}(\mathbb{Z})}$ and its associated norm \|\|$_{\ell_{p}^{2}(\mathbb{Z})}$ defined as follows: for $\mathcal{U}, \mathcal{V} \in \ell_{p}^{2}(\mathbb{Z})$

$$
<\mathcal{U}, \mathcal{V}>_{\ell_{p}^{2}(\mathbb{Z})}=\sum_{k \in \mathbb{Z}}\left(k^{2 p}+1\right)\left(\mathbf{u}_{k} \cdot \mathbf{v}_{k}\right) \quad \text { and } \quad\|\mathcal{U}\|_{\ell_{p}^{2}(\mathbb{Z})}^{2}=<\mathcal{U}, \mathcal{U}>_{\ell_{p}^{2}(\mathbb{Z})}
$$

We also introduce the space of fast decreasing series defined by $\ell_{\infty}^{2}(\mathbb{Z})=\bigcap_{p \in \mathbb{N}} \ell_{p}^{2}(\mathbb{Z})$.

Lemma 1. The spaces $\ell_{p}^{2}(\mathbb{Z})$ have the following properties.

1. $\ell_{0}^{2}(\mathbb{Z})=\ell^{2}(\mathbb{Z})$ and $\|\mathcal{U}\|_{\ell_{0}^{2}(\mathbb{Z})}=\sqrt{2}\|\mathcal{U}\|_{\ell^{2}(\mathbb{Z})}$ for all $\mathcal{U} \in \ell_{0}^{2}(\mathbb{Z})$.

2. The inclusion $\ell_{p}^{2}(\mathbb{Z}) \subset \ell_{q}^{2}(\mathbb{Z})$ holds for all $(p, q) \in\left(\mathbb{R}^{+}\right)^{2}$ with $p \geq q$ and we have $\|\mathcal{U}\|_{\ell_{q}^{2}(\mathbb{Z})} \leq$ $\|\mathcal{U}\|_{\ell_{p}^{2}(\mathbb{Z})}$ for all $\mathcal{U} \in \ell_{p}^{2}(\mathbb{Z})$.

3. The inclusion $\ell_{p}^{2}(\mathbb{Z}) \subset \ell^{1}(\mathbb{Z})$ holds for all $p>1$ and we have $\|\mathcal{U}\|_{\ell^{1}(\mathbb{Z})} \leq \sqrt{1+2 \zeta(2 p)}\|\mathcal{U}\|_{\ell_{p}^{2}(\mathbb{Z})}$ for all $\mathcal{U} \in \ell_{p}^{2}(\mathbb{Z})$ where $\zeta$ is the Riemann function.

4. If $\mathcal{S}$ denote the shift operator on $\ell^{2}(\mathbb{Z})$ defined by $(\mathcal{S U})_{k}=\mathbf{u}_{\mathbf{k}-\mathbf{1}}$ for all $k \in \mathbb{Z}$ then for all $p \in \mathbb{N}$ we have

$$
\|\mathcal{S U}\|_{\ell_{p}^{2}(\mathbb{Z})} \leq 2^{p}\|\mathcal{U}\|_{\ell_{p}^{2}(\mathbb{Z})} \quad \text { and } \quad\left\|\mathcal{S}^{-1} \mathcal{U}\right\|_{\ell_{p}^{2}(\mathbb{Z})} \leq 2^{p}\|\mathcal{U}\|_{\ell_{p}^{2}(\mathbb{Z})}
$$

Proof The first two properties are obvious consequences of the definition of the spaces $\ell_{p}^{2}(\mathbb{Z})$. The inclusion of $\ell_{p}^{2}(\mathbb{Z})$ into $\ell^{1}(\mathbb{Z})$ can be shown easily using the Cauchy-Schwarz inequality. The proof of the last inequalities reads:

$$
\begin{aligned}
\|\mathcal{S U}\|_{\ell_{p}^{2}(\mathbb{Z})}^{2} & =\sum_{k \in \mathbb{Z}}\left(k^{2 p}+1\right)\left|\mathbf{u}_{\mathbf{k}-\mathbf{1}}\right|^{2}=\sum_{k \in \mathbb{Z}}\left((k+1)^{2 p}+1\right)\left|\mathbf{u}_{k}\right|^{2}=\sum_{k \in \mathbb{Z}}\left(\sum_{n=0}^{2 p} C_{2 p}^{n} k^{n}+1\right)\left|\mathbf{u}_{k}\right|^{2} \\
& \leq \sum_{n=0}^{2 p} C_{2 p}^{n} \sum_{k \in \mathbb{Z}}\left(k^{n}+1\right)\left|\mathbf{u}_{k}\right|^{2} \leq \sum_{n=0}^{2 p} C_{2 p}^{n}\|\mathcal{U}\|_{\ell_{n / 2}^{2}(\mathbb{Z})}^{2} \leq 2^{2 p}\|\mathcal{U}\|_{\ell_{p}^{2}(\mathbb{Z})}^{2},
\end{aligned}
$$

with a similar argument for the second inequality. 
Eventually we define the space $L_{l o c}^{\infty}\left(\mathbb{R}^{+} ; \ell_{p}^{2}(\mathbb{Z})\right)$ of sequences of functions $\mathcal{U}: t \in \mathbb{R}^{+} \mapsto$ $\left(\mathbf{u}_{k}(t)\right)_{k \in \mathbb{Z}} \in \ell^{2}(\mathbb{Z})$ such that for all $t \in \mathbb{R}^{+}$we have $\left(\mathbf{u}_{k}(t)\right)_{k \in \mathbb{Z}} \in \ell_{p}^{2}(\mathbb{Z})$ and the space $\mathcal{C}\left(\mathbb{R}^{+} ; \ell_{\infty}^{2}(\mathbb{Z})\right)$ of sequences of functions such that for all $p \in \mathbb{N}$ the function $t \in \mathbb{R}^{+} \mapsto$ $\|\mathcal{U}(t)\|_{\ell_{p}^{2}(\mathbb{Z})}$ is continuous.

\subsection{Existence and uniqueness results for the sequence $\left(\mathbf{m}_{k}\right)_{k \in \mathbb{Z}}$}

The sequence of differential equations (23) can be written as an infinite differential system [21] for $\mathcal{M}=\left(\mathbf{m}_{k}(t)\right)_{k \in \mathbb{Z}}$ as follows

$$
\left\{\begin{array}{l}
\frac{\mathrm{d}}{\mathrm{d} t} \mathcal{M}(t)=\mathcal{A M}(t)+\mathcal{B} \\
\mathcal{M}(0)=\mathcal{M}_{0}
\end{array}\right.
$$

where the operator $\mathcal{A}$ is defined by $(\mathcal{A M})_{k}=A_{2} \mathbf{m}_{\mathbf{k}-\mathbf{1}}+A_{0} \mathbf{m}_{k}+A_{-2} \mathbf{m}_{\mathbf{k}+\mathbf{1}}-2 \mathrm{i} k \omega_{0} \mathbf{m}_{k}$ for all $k \in \mathbb{Z}$, and where $(\mathcal{B})_{k}=\delta_{k} \mathbf{b}$ and $\left(\mathcal{M}_{0}\right)_{k}=\delta_{k} \mathbf{M}_{\mathbf{0}}$.

We carry out the following change of unknown to remove the dependence on $k$ in the expression of $\mathcal{A}$ : for each $k \in \mathbb{Z}$ we set $\mathbf{u}_{k}: t \in \mathbb{R}^{+}+\mapsto e^{2 \mathrm{i} k \omega_{0} t} \mathbf{m}_{k}(t)$. Since $\left|\mathbf{u}_{k}(t)\right|=\left|\mathbf{m}_{k}(t)\right|$ the asymptotic behaviour of the sequences $\mathcal{U}=\left(\mathbf{u}_{k}(t)\right)_{k \in \mathbb{Z}}$ and $\mathcal{M}=\left(\mathbf{m}_{k}(t)\right)_{k \in \mathbb{Z}}$ when $k$ tends to infinity are similar. In terms of the new unknown $\mathcal{U}=\left(\mathbf{u}_{k}\right)_{k \in \mathbb{Z}}$ the sequence of differential equations (23) reads

$$
\forall k \in \mathbb{Z} \quad\left\{\begin{array}{l}
\frac{\mathrm{d}}{\mathrm{d} t} \mathbf{u}_{k}=A_{2} \mathbf{u}_{k-1} e^{2 \mathrm{i} \omega_{0} t}+A_{0} \mathbf{u}_{k}+A_{-2} \mathbf{u}_{k+1} e^{-2 \mathrm{i} \omega_{0} t}+\delta_{k} \mathbf{b} \\
\mathbf{u}_{k}(0)=\delta_{k} \mathbf{M}_{\mathbf{0}}
\end{array}\right.
$$

or equivalently

$$
\left\{\begin{array}{l}
\frac{\mathrm{d}}{\mathrm{d} t} \mathcal{U}(t)=\mathcal{A}_{s}(t) \mathcal{U}(t)+\mathcal{B} \\
\mathcal{U}(0)=\mathcal{M}_{0}
\end{array}\right.
$$

where now the linear operator $\mathcal{A}_{s}$ is time dependant and defined using the shift operator $\mathcal{S}$ and the identity $\mathcal{I}_{d}$ in $\ell^{2}(\mathbb{Z})$ as follows:

$$
\mathcal{A}_{s}(t)=A_{2} e^{2 \mathrm{i} \omega_{0} t} \mathcal{S}+A_{0} \mathcal{I}_{d}+A_{-2} e^{-2 \mathrm{i} \omega_{0} t} \mathcal{S}^{-1} .
$$

Using the estimate in lemma 1 for the shift operator $\mathcal{S}$ with $p=0$ we obtain that for all $\mathcal{Y} \in \ell^{2}(\mathbb{Z})$

$$
\left\|\mathcal{A}_{s}(t) \mathcal{Y}\right\|_{\ell^{2}(\mathbb{Z})} \leq\left(\left\|A_{2}\right\|+\left\|A_{0}\right\|+\left\|A_{-2}\right\|\right)\|\mathcal{Y}\|_{\ell^{2}(\mathbb{Z})}
$$

where || || denotes the matricial norm subordinated to the euclidian vector norm. This relation shows that the linear operator $\mathcal{A}_{s}(t)$ is continuous from $\ell^{2}(\mathbb{Z})$ into $\ell^{2}(\mathbb{Z})$.

Proposition 3.1. There exists a unique solution $\mathcal{U} \in \mathcal{C}^{1}\left(\mathbb{R}^{+} ; \ell_{\infty}^{2}(\mathbb{Z})\right)$ to the differential system (27). Moreover, for all $p \in \mathbb{N}$ and for all $t \in \mathbb{R}^{+}$, the solution to (27) satisfies

$$
\|\mathcal{U}(t)\|_{\ell_{p}^{2}(\mathbb{Z})} \leq e^{C_{p} t}\left|\mathbf{M}_{\mathbf{0}}\right|+\left(e^{C_{p} t}-1\right) \frac{|\mathbf{b}|}{C_{p}}
$$

where $C_{p}=2^{p+1}\left\|A_{2}\right\|+\left\|A_{0}\right\|$ is a constant. 
Proof To prove existence and uniqueness of the solution, we use Cauchy-Lipschitz theorem (see $[16][17])$. We have to prove that the operator $(t, \mathcal{Y}) \in \mathbb{R}^{+} \times \ell^{2}(\mathbb{Z}) \mapsto \mathcal{A}_{s}(t) \mathcal{Y}+\mathcal{B}$ is continuous with respect to $t$ and Lipschitz with respect to $\mathcal{Y}$. The latter condition is fulfilled from relation (29). Continuity with respect to $t$ is obvious from the definition of $\mathcal{A}_{s}$, see relation (28). As the initial data sequence satisfies $\mathbf{u}_{k}(0)=\delta_{k} \mathbf{M}_{\mathbf{0}}$, we have $\mathcal{U}(0) \in \ell^{2}(\mathbb{Z})$ and therefore Cauchy-Lipschitz theorem indicates that the solution $\mathcal{U}$ to the problem $(27)$ is unique and belongs to $\mathcal{C}^{1}\left(\mathbb{R}^{+} ; \ell^{2}(\mathbb{Z})\right)$.

Let us now prove the estimate (30). On the one hand, using relation (28) for the operator $\mathcal{A}_{s}(t)$ and the estimate in lemma 1 for the shift operator we obtain: $\forall p \in \mathbb{N} \quad \forall \mathcal{Y} \in \ell_{p}^{2}(\mathbb{Z})$

$$
\left\|\mathcal{A}_{s}(t) \mathcal{Y}\right\|_{\ell_{p}^{2}(\mathbb{Z})} \leq\left(2^{p}\left\|A_{2}\right\|+\left\|A_{0}\right\|+2^{p}\left\|A_{-2}\right\|\right)\|\mathcal{Y}\|_{\ell_{p}^{2}(\mathbb{Z})}=\left(2^{p+1}\left\|A_{2}\right\|+\left\|A_{0}\right\|\right)\|\mathcal{Y}\|_{\ell_{p}^{2}(\mathbb{Z})} .
$$

On the other hand, for $p \in \mathbb{N}$ and $t \in \mathbb{R}^{+}$we deduce from $(27)$ that $\mathcal{U}(t) \in \ell_{p}^{2}(\mathbb{Z})$ satisfies

$$
\frac{1}{2} \frac{\mathrm{d}}{\mathrm{d} t}\|\mathcal{U}(t)\|_{\ell_{p}^{2}(\mathbb{Z})}^{2}=<\frac{\mathrm{d}}{\mathrm{d} t} \mathcal{U}(t), \mathcal{U}(t)>_{\ell_{p}^{2}(\mathbb{Z})}=<\mathcal{A}_{s}(t) \mathcal{U}(t), \mathcal{U}(t)>_{\ell_{p}^{2}(\mathbb{Z})}+<\mathcal{B}, \mathcal{U}(t)>_{\ell_{p}^{2}(\mathbb{Z})} .
$$

Then, from the Cauchy-Schwarz inequality we have

$$
\|\mathcal{U}(t)\|_{\ell_{p}^{2}(\mathbb{Z})} \frac{\mathrm{d}}{\mathrm{d} t}\|\mathcal{U}(t)\|_{\ell_{p}^{2}(\mathbb{Z})} \leq\left\|\mathcal{A}_{s}(t) \mathcal{U}(t)\right\|_{\ell_{p}^{2}(\mathbb{Z})}\|\mathcal{U}(t)\|_{\ell_{p}^{2}(\mathbb{Z})}+\|\mathcal{B}\|_{\ell_{p}^{2}(\mathbb{Z})}\|\mathcal{U}(t)\|_{\ell_{p}^{2}(\mathbb{Z})} .
$$

Now, from (31) we obtain

$$
\frac{\mathrm{d}}{\mathrm{d} t}\|\mathcal{U}(t)\|_{\ell_{p}^{2}(\mathbb{Z})} \leq C_{p}\|\mathcal{U}(t)\|_{\ell_{p}^{2}(\mathbb{Z})}+\|\mathcal{B}\|_{\ell_{p}^{2}(\mathbb{Z})}
$$

Since $\|\mathcal{U}(0)\|_{\ell_{p}^{2}(\mathbb{Z})}=\left|\mathbf{M}_{\mathbf{0}}\right|$ and $\|\mathcal{B}\|_{\ell_{p}^{2}(\mathbb{Z})}=|\mathbf{b}|$, Gronwall lemma (see [16][17]) gives the estimate

$$
\|\mathcal{U}(t)\|_{\ell_{p}^{2}(\mathbb{Z})} \leq e^{C_{p} t}\left|\mathbf{M}_{\mathbf{0}}\right|+\left(e^{C_{p} t}-1\right) \frac{|\mathbf{b}|}{C_{p}}
$$

The proof is completed since we can now assert that the solution $\mathcal{U}$ to the problem (27) belongs to $\mathcal{C}^{1}\left(\mathbb{R}^{+} ; \ell_{\infty}^{2}(\mathbb{Z})\right)$.

Corollary 1. There exists a unique solution $\left(\mathbf{m}_{k}\right)_{k \in \mathbb{Z}}$ to the system of differential equations (23); it satisfies

$$
\forall t \in \mathbb{R}^{+}+\forall p \in \mathbb{N} \quad\left(\mathbf{m}_{k}(t)\right)_{k \in \mathbb{Z}} \in \ell_{p}^{2}(\mathbb{Z}),
$$

i.e. $\mathbf{m}_{k}(t)$ tends towards zero as $k$ tends to $\pm \infty$ faster than any power of $1 / k$.

Proof It is a consequence of the definition of the functional space $\mathcal{C}^{1}\left(\mathbb{R} ; \ell_{\infty}^{2}(\mathbb{Z})\right)$ and the fact that the two sequences $\left(\mathbf{u}_{k}\right)_{k \in \mathbb{Z}}$ and $\left(\mathbf{m}_{k}\right)_{k \in \mathbb{Z}}$ satisfy $\left|\mathbf{u}_{k}\right|=\left|\mathbf{m}_{k}\right|$.

\subsection{Asymptotic behaviour of the sequence $\left(\mathbf{m}_{k}\right)_{k \in \mathbb{Z}}$}

As the sequence $\left(\mathbf{u}_{k}(t)\right)_{k \in \mathbb{Z}}$ tends very quickly towards zero as $k$ tends to $\pm \infty$, an approximation of the values of $\mathbf{u}_{k}$ can be obtained by computing the solution $\mathcal{U}^{[N]}=\left(\mathbf{u}_{k}^{[N]}\right)_{k \in \mathbb{Z}}$ of the following finite system of differential equations deduced from (26) by truncation:

$$
\left\{\begin{array}{l}
\frac{\mathrm{d}}{\mathrm{d} t} \mathbf{u}_{k}^{[N]}(t)=A_{2} e^{2 \mathrm{i} \omega_{0} t} \mathbf{u}_{k-1}^{[N]}(t)+A_{0} \mathbf{u}_{k}^{[N]}(t)+A_{-2} e^{-2 \mathrm{i} \omega_{0} t} \mathbf{u}_{k+1}^{[N]}(t)+\delta_{k} \mathbf{b}, \quad \forall|k| \leq N \\
\mathbf{u}_{k}^{[N]}=\mathbf{0}, \quad \forall|k|>N
\end{array}\right.
$$


under the initial condition $\mathcal{U}^{[N]}(0)=\mathcal{M}_{0}$. According to Cauchy-Lipschitz theorem, the finite system of differential equations (32) has a unique solution $\mathcal{U}^{[N]} \in \mathcal{C}^{1}\left(\mathbb{R}^{+}, \ell_{\infty}^{2}(\mathbb{Z})\right)$ since $\mathbf{u}_{\mathbf{k}}^{\mathbf{N}}=0$ for $|k|>N$.

The next proposition shows that $\mathcal{U}^{[N]}$ is a "good approximation" of the solution $\mathcal{U}$ of (26) for values of $N$ which are large enough.

Proposition 3.2. The sequence of functions $\left(\mathcal{E}^{[N]}\right)_{N \in \mathbb{N}}$ where $\mathcal{E}^{[N]}: t \in \mathbb{R}^{+} \mapsto \mathcal{U}(t)-\mathcal{U}^{[N]}(t)$ belongs to $L_{\text {loc }}^{\infty}\left(\mathbb{R}^{+} ; \ell_{\infty}^{2}\left(\mathbb{N}, \ell_{\infty}^{2}(\mathbb{Z})\right)\right)$. This means that for any $t \in \mathbb{R}^{+}$, the sequence of complex vectors $\left(\mathcal{E}^{[N]}(t)\right)_{N \in \mathbb{N}}$ tends towards zero faster than any power of $1 / N$. In other words, for all $t \in \mathbb{R}^{+}$the sequence $\mathcal{U}^{[N]}(t)$ converge very quickly to $\mathcal{U}(t)$ as $N$ tends to infinity.

Proof From equations (32) and (26) we deduce that $\mathcal{E}^{[N]}=\left(\mathbf{e}_{\mathbf{k}}^{[\mathbf{N}]}\right)_{k \in \mathbb{Z}} \in \mathcal{C}^{1}\left(\mathbb{R}^{+}, \ell_{\infty}^{2}(\mathbb{Z})\right)$ satisfies the following infinite system of differential equations

$$
\left\{\begin{array}{l}
\frac{\mathrm{d}}{\mathrm{d} t} \mathbf{e}_{k}^{[N]}(t)=A_{2} e^{2 \mathrm{i} \omega_{0} t} \mathbf{e}_{k-1}^{[N]}+A_{0} \mathbf{e}_{k}^{[N]}+A_{-2} e^{-2 \mathrm{i} \omega_{0} t} \mathbf{e}_{k+1}^{[N]}, \quad \forall|k| \neq N+1 \\
\frac{\mathrm{d}}{\mathrm{d} t} \mathbf{e}_{N+1}^{[N]}(t)=A_{2} e^{2 \mathrm{i} \omega_{0} t} \mathbf{u}_{N}+A_{0} \mathbf{e}_{N+1}^{[N]}+A_{-2} e^{-2 \mathrm{i} \omega_{0} t} \mathbf{e}_{N+2}^{[N]} \\
\frac{\mathrm{d}}{\mathrm{d} t} \mathbf{e}_{-N-1}^{[N]}(t)=A_{2} e^{2 \mathrm{i} \omega_{0} t} \mathbf{e}_{-N-2}^{[N]}+A_{0} \mathbf{e}_{-N-1}^{[N]}+A_{-2} e^{-2 \mathrm{i} \omega_{0} t} \mathbf{u}_{-N}
\end{array}\right.
$$

under the initial condition $\mathcal{E}^{[N]}(0)=0$.

To get an estimate of $\mathcal{E}^{[N]}(t)$ for $t \in \mathbb{R}^{+}$we proceed on a similar manner as the one used in the proof of proposition 3.1. Considering the scalar-product by $\mathbf{e}_{k}^{[N]}$ of each side of the $k$ th equation in (33) and summing over $k \in \mathbb{Z}$ we get

$\frac{1}{2} \frac{\mathrm{d}}{\mathrm{d} t}\left(\left\|\mathcal{E}^{[N]}\right\|_{\ell_{p}^{2}(\mathbb{Z})}^{2}(t)\right) \leq C_{p}\left\|\mathcal{E}^{[N]}(t)\right\|_{\ell_{p}^{2}(\mathbb{Z})}^{2}+\left|A_{2} e^{2 \mathrm{i} \omega_{0} t}\left(\mathbf{u}_{N} \cdot \mathbf{e}_{N+1}^{[N]}\right)\right|+\left|A_{-2} e^{-2 \mathrm{i} \omega_{0} t}\left(\mathbf{u}_{-N} \cdot \mathbf{e}_{-N-1}^{[N]}\right)\right|$.

We use the fact that $\mathbf{u}_{N}=\overline{\mathbf{u}_{-N}}$ to deduce that

$$
\frac{\mathrm{d}}{\mathrm{d} t}\left(\left\|\mathcal{E}^{[N]}\right\|_{\ell_{p}^{2}(\mathbb{Z})}^{2}(t)\right) \leq 2\left(C_{p}+\left\|A_{2}\right\|\right)\left\|\mathcal{E}^{[N]}\right\|_{\ell_{p}^{2}(\mathbb{Z})}^{2}(t)+2\left\|A_{2}\right\|\left|\mathbf{u}_{N}(t)\right|^{2} .
$$

Multiplying each side of this estimate by $N^{2 q}+1, q \in \mathbb{N}$ and summing over all $N \in \mathbb{Z}$ we have

$$
\frac{\mathrm{d}}{\mathrm{d} t} F(t) \leq 2\left(C_{p}+\left\|A_{2}\right\|\right) F(t)+2\left\|A_{2}\right\|\|\mathcal{U}(t)\|_{\ell_{q}^{2}(\mathbb{Z})}^{2},
$$

where $F(t)=\sum_{N \in \mathbb{N}}\left(N^{2 q}+1\right)\left\|\mathcal{E}^{[N]}(t)\right\|_{\ell_{p}^{2}(\mathbb{Z})}$ is the square of the $\ell_{q}^{2}(\mathbb{Z})$-norm of the sequence $\left\|\mathcal{E}^{[N]}(t)\right\|_{\ell_{p}^{2}(\mathbb{Z})}$. Finally, Gronwall lemma and the estimate for $\mathcal{U}$ given in proposition 3.1 show that for all $t \in \mathbb{R}^{+}$

$$
\begin{aligned}
F(t) & \leq 2\left\|A_{2}\right\| \int_{0}^{T} e^{2\left(C_{p}+\left\|A_{2}\right\|\right)(T-s)}\|\mathcal{U}\|_{\ell_{q}^{2}(\mathbb{Z})}^{2}(s) d s \\
& \leq 2\left\|A_{2}\right\| \int_{0}^{T} e^{2\left(C_{p}+\left\|A_{2}\right\|\right)(T-s)}\left(e^{C_{p} s}\left|\mathbf{M}_{\mathbf{0}}\right|+\left(e^{C_{p} s}-1\right) \frac{|\mathbf{b}|}{C_{p}}\right)^{2} d s .
\end{aligned}
$$

The proof is completed since for all time $t \in \mathbb{R}^{+}$we have shown that the sequence $\left(\left(\mathcal{E}_{k}^{[N]}(t)\right)_{k \in \mathbb{Z}}\right)_{N \in \mathbb{N}}$ is bounded in $\ell_{q}^{2}\left(\mathbb{N}, \ell_{p}^{2}(\mathbb{Z})\right)$ for all $p$ and $q$. 
To conclude this section it can be notice that, as a consequence of proposition 3.2, the sequence $\mathcal{M}^{[N]}=\left(\mathbf{m}_{k}^{[N]}\right)_{k \in \mathbb{Z}}$ defined by

$$
\forall k \in \mathbb{Z} \quad \forall N \in \mathbb{N} \quad \forall t \in \mathbb{R}^{+} \quad \mathbf{m}_{k}^{[N]}(t)=e^{-2 \mathrm{i} k \omega_{0} t} \mathbf{u}_{k}^{[N]}(t)
$$

will be a good approximation of the solution $\mathcal{M}$ to the system (25) for values of $N$ which are large enough.

\section{Numerical approximation of the sequence $\left(\mathbf{m}_{\mathbf{k}}\right)_{k \in \mathbb{Z}}$}

\subsection{The algorithm}

The differential system (25) can be seen as the following infinite matricial differential system:

$$
\frac{\mathrm{d}}{\mathrm{d} t} \mathcal{M}(t)=\mathcal{A M}(t)+\mathcal{B}
$$

where $\mathcal{M}(t)=\left(\mathbf{m}_{k}(t)\right)_{k \in \mathbb{Z}}$ and $\mathcal{B}=\left(\mathbf{b}_{k}\right)_{k \in \mathbb{Z}}$ are infinite vectors and $\mathcal{A}$ is the constant infinite matrix defined by

$$
\mathcal{A}=\left(\begin{array}{ccccccc}
\ddots & \ddots & \ddots & & & & \\
& A_{2} & A_{0}(1) & A_{-2} & & & \\
& & A_{2} & A_{0} & A_{-2} & & \\
& & & A_{2} & A_{0}(-1) & A_{-2} & \\
& & & & \ddots & \ddots & \ddots
\end{array}\right)
$$

where for all $k \in \mathbb{Z}$ we have set $A_{0}(k)=A_{0}+2 \mathrm{i} k \omega_{0} \mathrm{Id}$.

As the sequence $\left(\mathbf{m}_{k}\right)_{k}$ tends very quickly towards $\mathbf{0}$ (see corollary 1 ) an approximation of the solution of the infinite differential system (35) can be obtained from the solution to the following finite differential system deduced from (35) by truncating the matrix $\mathcal{A}$ :

$$
\frac{\mathrm{d}}{\mathrm{d} t} \mathcal{M}^{[N]}(t)=\mathcal{A}^{[N]} \mathcal{M}^{[N]}(t)+\mathcal{B}^{[N]}
$$

where $\mathcal{A}^{[N]}$ is the constant matrix of size $3(2 N+1)$ defined by

$$
\mathcal{A}^{[N]}=\left(\begin{array}{ccccc}
A_{0}(N) & A_{-2} & & & \\
A_{2} & A_{0}(N-1) & A_{-2} & & \\
& \ddots & \ddots & \ddots & \\
& & \ddots & \ddots & A_{-2} \\
& & & A_{2} & A_{0}(-N)
\end{array}\right)
$$

and $\mathcal{B}^{[N]}=\left(\mathbf{b}_{k}\right)_{k \in\{-N, \ldots, N\}}$. The solution to (36) under the initial condition $\mathcal{M}^{[N]}(0)=$ $\left(0, \ldots, 0, \mathbf{M}_{\mathbf{0}}, 0, \ldots, 0\right)$ is given by the Duhamel formula, see [16][17]:

$$
\mathcal{M}^{[N]}(t)=e^{t \mathcal{A}^{[N]}} \mathcal{M}^{[N]}(0)+\left(\int_{0}^{t} e^{(t-s) \mathcal{A}^{[N]}} d s\right) \mathcal{B}^{[N]} .
$$


If the matrix $\mathcal{A}^{[N]}$ is diagonalisable with $\mathcal{A}^{[N]}=P D P^{-1}$ and $\mathcal{D}=\operatorname{diag}\left(d_{i}, i=-3 N, \ldots, 3 N\right)$ then we have

$$
\mathcal{M}^{[N]}(t)=\mathcal{P} e^{t D} P^{-1} \mathcal{M}^{[N]}(0)+P \mathcal{S}(t) P^{-1} \mathcal{B}^{[N]}
$$

where $\mathcal{S}(t)=\int_{0}^{t} e^{(t-s) D} d s=\operatorname{diag}\left(s_{i}(t), i=1, \ldots, N\right)$ and $s_{i}(t)=\left(\mathrm{e}^{t d_{i}}-1\right) / d_{i}$ if $d_{i} \neq 0$ and $s_{i}(t)=t$ otherwise.

The algorithm to compute an approximation of the solution to the Bloch equation for a given volume element (voxel) is therefore the following.

1. Chose the truncation order $N$ and duration $T_{R F}$ of the RF pulse.

2. Compute the eigenvalues and eigenvectors of the block tridiagonal matrix $\mathcal{A}^{[N]}$ as given by (37).

3. Form the matrices $P$ and $D$ from the eigenvalues and eigenvectors.

4. Compute the $s_{i}$ 's from the eigenvalues.

5. Compute the inverse of $P$.

6. Compute $\mathcal{M}^{[N]}\left(T_{R F}\right)=\left(\mathbf{m}_{k}\left(T_{R F}\right)\right)_{k \in\{-N, \ldots, N\}}$ from relation (39)

$$
\mathcal{M}^{[N]}\left(T_{R F}\right)=P e^{T_{R F} D} P^{-1} \mathcal{M}^{[N]}(0)+P \mathcal{S}\left(T_{R F}\right) P^{-1} \mathcal{B}^{[N]} .
$$

7. Compute the magnetisation vector in the rotating frame using the following formula deduced from $(22)$ :

$$
\mathbf{m}\left(T_{R F}\right) \approx \sum_{k=-N}^{N} \mathbf{m}_{k}\left(T_{R F}\right) e^{2 \mathrm{i} k \omega_{0} T_{R F}}
$$

where $\mathbf{m}_{\mathbf{k}}(T)$ is given by the components $3(N+k)+1$ to $3(N+k)+3$ of the vector $\mathcal{M}^{[N]}(T)$ of size $3(2 N+1)$.

8. Compute the approximate magnetisation vector in the laboratory frame :

$$
\mathbf{M}^{[N]}\left(T_{R F}\right)=R\left(T_{R F}\right) \mathbf{m}^{[N]}\left(T_{R F}\right)
$$

where $R\left(T_{R F}\right)$ is the rotation matrix given by $(7)$.

Remark 1. In this algorithm, the cost in computational time lies in the computation of the eigenvalues and eigenvectors of the matrix $\mathcal{A}^{[N]}$. This computation can be achieved very quickly if adequate computation algorithm for tridiagonal matrices are used [22].

2. When a full MRI sequence is used, only the initial conditions for the Bloch equation are changing from on pulse to the other. The matrix of the differential system remains unchanged and therefore to handle a complete MRI sequence, a loop on steps 6 to 8 is only required. Indeed, as the matrix $\mathcal{A}^{[N]}$ remains unchanged during the whole MRI sequence, there is no need to compute its eigen-elements for each pulse.

3. In standard MRI experiments the values of the diagonal elements $\tau_{1}$ and $\tau_{2}$ are small compare to the others elements in the matrix $\mathcal{A}^{[N]}$. Let us decompose the matrix $\mathcal{A}^{[N]}$ as $\mathcal{A}^{[N]}=\mathcal{H}^{[N]}+\mathcal{F}^{[N]}$ where $\mathcal{F}^{[N]}=\operatorname{diag}\left(-\tau_{2},-\tau_{2},-\tau_{1}, \ldots,-\tau_{2},-\tau_{2},-\tau_{1}\right)$ and $\mathcal{H}^{[N]}$ is a skew-hermitian matrix since $A_{-2}^{*}=-A_{2}$. The eigenvalues of $\mathcal{H}^{[N]}$ are all pure imaginary numbers and there exists a unitary matrix $\mathcal{Q}^{[N]}$ and a diagonal matrix $\mathcal{D}^{[N]}$ such that $\mathcal{H}^{[N]}=\mathcal{Q}^{[N]} \mathcal{D}^{[N]} \mathcal{Q}^{[N]^{*}}$. It follows from perturbation theory for hermitian matrices [23] that if $d_{i}$ is an eigenvalue of $\mathcal{A}^{[N]}$ then there exists some eigenvalue $\tilde{d}_{i}$ of $\mathcal{H}^{[N]}$ for which

$$
\left|d_{i}-\tilde{d}_{i}\right| \leq\left\|\mathcal{F}^{[N]}\right\|_{2} .
$$


Moreover, from Davis and Kahan's theorem [23], if $\left(\tilde{d}_{i}, \tilde{v}_{i}\right)$ is an approximation of the eigenpair $\left(d_{i}, v_{i}\right)$ and $\delta$ is the gap between $\tilde{d}_{i}$ and any other eigenvalue then we have the following estimate

$$
\left|d_{i}-\tilde{d}_{i}\right| \leq \frac{\|r\|_{2}^{2}}{\delta} \quad \text { and } \quad \sin \angle\left(v_{i}, \tilde{v}_{i}\right) \leq \frac{\|r\|_{2}}{\delta} .
$$

where $\sin \angle\left(v_{i}, \tilde{v}_{i}\right)$ is the angle between $\tilde{v}_{i}$ and the eigenspace corresponding to those eigenvalues closest to $\tilde{d}_{i}$ and $r$ is the following residual $r=\mathcal{H}^{[N]} \tilde{v}_{i}-\tilde{d}_{i} \tilde{v}_{i}$. As a consequence, in a standard MRI experiment the following approximation can be done: $\mathcal{A}^{[N]} \approx \mathcal{H}^{[N]}$. This approximation improve the efficiency of the algorithm since we have to compute the eigen-elements of a skewhermitian matrix and $P^{-1}=P^{*}$.

\subsection{An overview of accuracy and efficiency of the method}

In order to get information on its accuracy we have compared the values of the magnetisation given by our method to the exact solution of the Bloch equation (available only when no RF perturbation exists) and to the solution computed by the ode 45 solver of MATLAB (all with default accuracy values). The computations were done on a Intel Pentium IV 3 Ghz personal computer under matlaB. The following values were used : $B_{0}=1$ Telsa, $B_{1}=10^{-3}$ Tesla, $\eta_{0}=1, T_{1}=750 \mathrm{~ms}, T_{2}=50 \mathrm{~ms}$ and $\theta=\frac{\pi}{2}$. Comparison of accuracy and cpu times for $16^{3}=4096$ executions is given in Table $\mathrm{I}$.

Table I. Comparison of accuracy and cpu times (in s.) for $16^{3}$ executions using the method with different values of $N$ and the ode45 solver.

\begin{tabular}{|c|c|c|c|c|c|}
\hline & $N=0$ & $N=1$ & $N=2$ & $N=3$ & ode45 \\
\hline CPU times & 3.82 & 9.17 & 17.56 & 29.53 & 69.21 \\
\hline Error in\% & 0.0089 & 0.0089 & 0.0089 & 0.0089 & 0.01 \\
\hline
\end{tabular}

When RF perturbations are involved, the Bloch equation does not possesses an exact solution. In table 4.2 we have compared, for different truncation orders $N$, the values of the magnetisation and the CPU times. We have considered a random RF perturbation of same magnitude as the RF field $\mathbf{B}_{\mathbf{1}}$. Computation with the ode45 MATLAB solver gives the following approximate solution $\mathbf{M}=(-0.22511588898239,0.97495030267178,-0.00903991786250)$; computation CPU time is 1325.18 s. It can be see that a good approximation of the solution

Table II. Comparison of solutions and CPU times (in s.) for $16^{3}$ executions using the method with different values of $N$.

\begin{tabular}{|c|c|c|c|c|}
\hline & $N=0$ & $N=1$ & $N=2$ & $N=3$ \\
\hline Times & 4.36 & 12.1 & 25.97 & 47.8 \\
\hline \multirow{3}{*}{$\mathbf{M}$} & -0.24326287201152 & -0.24326289631217 & -0.24326289631192 & -0.24326289631189 \\
& 0.96630713580637 & 0.96630713685089 & 0.96630713685049 & 0.96630713685088 \\
& 0.07993334331631 & 0.07993336879906 & 0.07993336879932 & 0.07993336879911 \\
\hline
\end{tabular}


is already obtained for $N=0$ and that the convergence of the method is very fast. The exponential convergence of the method has been explained in section 3. However, the fact that a good approximation of the solution is obtained for $N=0$ for usual values of the parameter used in MRI can be surprising. Let us explore the underlying reasons. For $N=0$ the approximation of $\mathbf{m}(t)$ is $\mathbf{m}^{[0]}(t)=\mathcal{M}_{0}(t)$ and from equation (36) we have $\mathbf{m}^{[0]}$ solution of the differential system

$$
\frac{\mathrm{d}}{\mathrm{d} t} \mathbf{m}^{[0]}(t)=\mathcal{A}_{0} \mathbf{m}^{[0]}(t)+\mathcal{B}_{0}=A_{0} \mathbf{m}^{[0]}(t)+\mathbf{b}
$$

where $A_{0}$ is given by $(21)$, whereas $\mathbf{m}(t)$ is solution of the differential system

$$
\frac{\mathrm{d}}{\mathrm{d} t} \mathbf{m}(t)=\tilde{A}(t) \mathbf{m}(t)+\mathbf{b}
$$

under an identical initial condition $\mathbf{m}(0)=\mathbf{m}^{[0]}(0)=\mathbf{M}_{\mathbf{0}}$. For the two differential equations (41) and (42), we have the integral representation formula:

$$
\mathbf{m}(t)=\mathbf{M}_{\mathbf{0}}+\int_{0}^{t} \tilde{A}(s) \mathbf{m}(s) \mathrm{d} s+\mathbf{b} t \quad \text { and } \quad \mathbf{m}^{[0]}(t)=\mathbf{M}_{\mathbf{0}}+A_{0} \int_{0}^{t} \mathbf{m}^{[0]}(s) \mathrm{d} s+\mathbf{b} t .
$$

From the Fourier series expansion of $\tilde{A}=A_{0}+\mathrm{e}^{-\mathrm{i} 2 \omega_{0} s} A_{2}+\mathrm{e}^{\mathrm{i} 2 \omega_{0} s} A_{-2}$ we deduce that

$$
\mathbf{m}(t)=\mathbf{m}^{[0]}(t)+\int_{0}^{t} \mathrm{e}^{-\mathrm{i} 2 \omega_{0} s} A_{2} \mathbf{m}(s) \mathrm{d} s+\int_{0}^{t} \mathrm{e}^{\mathrm{i} 2 \omega_{0} s} A_{-2} \mathbf{m}(s) \mathrm{d} s
$$

and integration by parts shows that

$$
\int_{0}^{t} \mathrm{e}^{ \pm \mathrm{i} 2 \omega_{0} s} A_{\mp 2} \mathbf{m}(s) \mathrm{d} s=\mathcal{O}\left(a_{2} \omega_{0}^{-1}\right)
$$

where $a_{2}=\left\|A_{2}\right\|=\left\|A_{-2}\right\|=\mathcal{O}\left(\omega_{1}\right)$. It follows that the difference between the 2 vectors $\mathbf{m}^{[0]}(t)$ and $\mathbf{m}(t)$ satisfies $\mathbf{y}^{\prime}(t)=A_{0} \mathbf{y}(t)+\mathcal{O}\left(\omega_{1} / \omega_{0}\right)$ under the initial condition $\mathbf{y}(0)=\mathbf{0}$. Therefore we have $\mathbf{m}(t)=\mathbf{m}^{[0]}(t)+\mathcal{O}\left(\omega_{1} / \omega_{0}\right)$. It means that when the static magnetic field $\mathbf{B}_{\mathbf{0}}$ has sufficiently large values or the $\mathrm{RF}$ field $\mathbf{B}_{\mathbf{1}}$ has sufficiently small values the quantity $\mathbf{m}^{[0]}(t)$ is a good approximation of $\mathbf{m}(t)$. Typically in a MRI experiment the field $\mathbf{B}_{\mathbf{0}}$ is around 1 Tesla whereas $\mathbf{B}_{\mathbf{1}}$ is around $10^{-3}$ Tesla so that $\omega_{1} / \omega_{0} \approx 10^{-3}$.

\subsection{Description of the magnetisation vector evolution}

As seen in section 2 the evolution of the magnetisation in the rotating frame $\left(\mathbf{e}_{\mathbf{1}}, \mathbf{e}_{\mathbf{2}}, \mathbf{e}_{\mathbf{3}}\right)$ without RF perturbation is described by relation (15). The magnetisation $\mathbf{m}$ starts to flip out of the $\mathbf{z}$ axis from its equilibrium position $\mathbf{M}_{\mathbf{0}}=\eta_{0} \mathbf{z}$ under the influence of the RF field $\mathbf{B}_{\mathbf{1}}$. The magnetisation $\mathbf{m}$ evolves in a plane perpendicular to $\mathbf{e}_{\mathbf{1}}$. After a length of time $T_{R F}=\frac{\pi}{2 \omega_{1}}$, the magnetisation vector rotates 90 degrees and lies in the transverse plane $(\mathbf{x}, \mathbf{y})$, see Fig. 1. It is in this position that it can be detected giving rise to the MRI signal during the relaxation process. View from the laboratory frame, the magetisation vector evolves in a spiroidal movement, see the right picture in Fig. 1.

The evolution of the magnetisation vector $\mathbf{m}$ is modified under RF perturbations. We have seen in section 4.2 that the solution $\mathbf{m}^{[0]}$ of the differential system

$$
\frac{\mathrm{d}}{\mathrm{d} t} \mathbf{m}^{[0]}(t)=A_{0} \mathbf{m}^{[0]}(t)+\mathbf{b}
$$



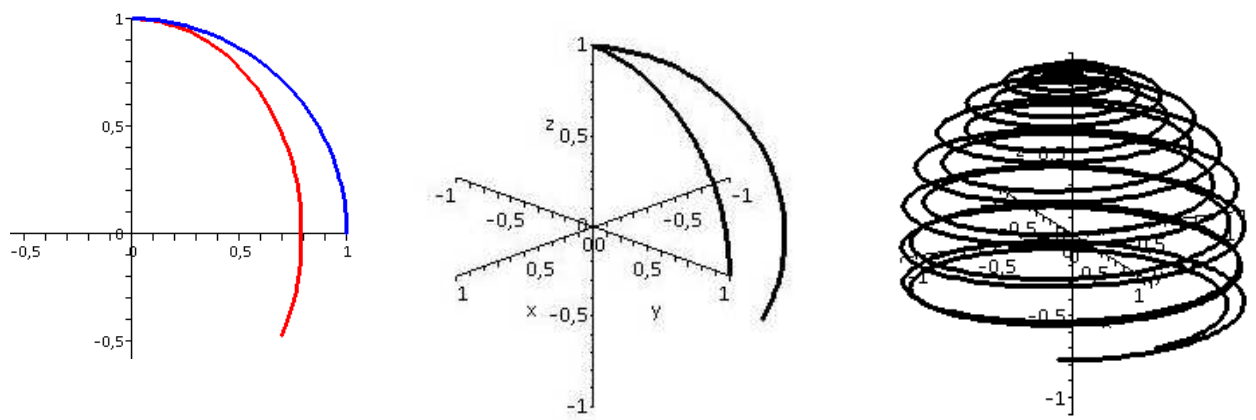

Figure 1. Comparison of the magnetisation vector evolution for a $\pi / 2$ pulse with and without $\mathrm{RF}$ perturbation: from left to right : in the plane $\left(\mathbf{e}_{\mathbf{2}}, \mathbf{z}\right)$, in the rotating frame $\left(\mathbf{e}_{\mathbf{1}}, \mathbf{e}_{\mathbf{2}}, \mathbf{z}\right)$ and in the laboratory frame $(\mathbf{x}, \mathbf{y}, \mathbf{z})$.

where $A_{0}$ is given by (21) can be considered as a good approximation of $\mathbf{m}$. The solution to this linear differential equation with constant coefficients can be computed analytically. Under the assumption that $\tau_{1}$ and $\tau_{2}$ can be neglected compared to $\omega_{1}$ the solution $\mathbf{m}^{[0]}$ to (43) under the initial condition $\mathbf{M}_{\mathbf{0}}=\eta_{0} \mathbf{z}$ reads

$$
\mathbf{m}^{[0]}(t)=\left(\begin{array}{c}
-\frac{v_{1}+u_{2}}{b} \eta_{0} \sin (\alpha t) \\
\frac{2 B_{1}+u_{1}-v_{2}}{b} \eta_{0} \sin (\alpha t) \\
M_{0} \cos (\alpha t)
\end{array}\right)
$$

where $b=\sqrt{\left(2 B_{1}+u_{1}-v_{2}\right)^{2}+\left(v_{1}+u_{2}\right)^{2}}$ and $\alpha=\frac{1}{2} \gamma b$. In Fig. 1 we have depicted together the evolution of the magnetisation vector with and without RF perturbations in the rotating frame $\left(\mathbf{e}_{\mathbf{1}}, \mathbf{e}_{\mathbf{2}}, \mathbf{e}_{\mathbf{3}}\right)$ and in the laboratory frame $(\mathbf{x}, \mathbf{y}, \mathbf{z})$ for a $\pi / 2$ pulse with $B_{1}=10^{-3}$ Tesla, $u_{1}=9.110^{-4}$ Tesla, $u_{2}=1.110^{-3}$ Tesla, $v_{1}=510^{-4}$ Tesla and $u_{2}=8.310^{-4}$ Tesla.

It can be seen from relation (44) and from Fig. 1 that under RF perturbation the magnetisation vector doesn't lie in the transverse $\mathbf{x}-\mathbf{y}$ plane after a length of time $T_{R F}=\frac{\pi}{2 \omega_{1}}$ as it could. The consequence is a diminution of the MRI signal intensity from normality and therefore an inaccurate MRI image.

\subsection{MATLAB program}

On the basis of this work and the one we devoted to susceptibility artifacts, see [6], we have developped a MATLAB program that simulates both the susceptibility and the eddy current artifacts in the case of a metallic implant with spherical shape (for such geometry, analytical expressions for the induced RF and static magnetic fields are known, see [24]). These two kinds of artifact add to the MRI image without our knowing of the contribution of each one to the image distortion. Our program enables to hide one of the two artifacts and image the other or image the effects of the two artifacts together. It is then possible to compare for different materials the importance of each source of image distorsion. The program can be obtained from the authors. 

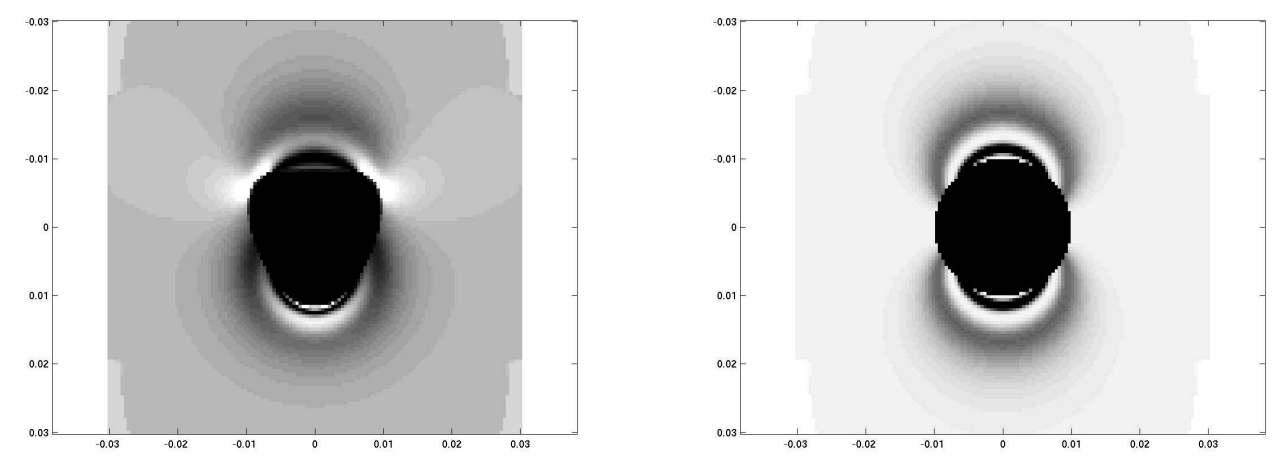

Figure 2. Simulation of the RF and the susceptibility artefacts generated by a ball (on the left) and visualisation of the RF artifact counterpart alone.

In Fig. 2 we show the artifact generated by a metallic ball made of copper with a magnetic susceptibility of $-210^{-5}$ usi and an electric conductivity of $5.910^{7}$. It is placed in a 2.5 Tesla magnetic field $\mathbf{B}_{\mathbf{0}}$ and a RF field $\mathbf{B}_{\mathbf{1}}$ of $10^{-3}$ Tesla. The slice depicted in Fig. 2 passes through the centre of the slice and is perpendicular to the magnetic field $\mathbf{B}_{\mathbf{0}}$. It has a side length of $5 \mathrm{~cm}$. The slice-selection gradient and the read-out gradient strength are $10^{-2} \mathrm{Tesla} / \mathrm{meter}$. The slice thickness is $3 \mathrm{~mm}$. Comparison of the two images shows the contribution of RF field perturbation to the image artifact (the non disturbed image should be a black disk in a uniformly grey background).

\section{Conclusion}

We have presented a method to solve numerically the Bloch equation under RF field inhomogeneities and we have justified it from a mathematical point of view. The method is based on a truncated series expansion of the solution and it is suited to furnish directly the position of the magnetisation vector at the end of the resonance process at time $T$ without having to compute it successively at the nodes of a subdivision of the time interval $[0, T]$. The computational cost of the method reduces to the computation of the eigen-elements of a block tridiagonal matrix of very small size (usually $3 \times 3$ or $9 \times 9$ ). Although the method has been designed to solve the Bloch equation in the context of MRI artifact simulation, it has a more general range of use. The method is devoted to be implemented in MRI simulator (such as the SIMRI project [25]) in order to take into account RF effects in the imaging process and enable the simulation of RF artifact induced by objects of very general shapes under a large variety of image acquisition sequences. 


\section{REFERENCES}

1. C.P. Slichter, editor. Principles of Magnetic Resonance. Springer-Verlag, New-York, 1990.

2. P.T. Callaghan. Principles of Nuclear Magnetic Resonance Microscopy. Oxford University Press,, 1994.

3. M.G. Abele, H. Rusinek, F. Bertora, and A. Trequattrini. Compensation of field distortion with ferromagnetic material and permanent magnets. Journal of Applied Physics, 75(10):6990-699, 1994.

4. A. Alexander, J. Tsuruda, and D. Parker. Elimination of Eddy current artifacts in diffusion weighted Echo Planar Images: the use of bipolar gradients. Journal of Magnetic Resonance in Medicine, 38:1016-1021, 1997.

5. J.F. Schenck. The role of magnetic susceptibility in magnetic resonance imaging. Med. Phys., 23(6):815850, 1996.

6. S. Balac and G. Caloz. Mathematical modeling and numerical simulation of magnetic susceptibility artifacts in Magnetic Resonance Imaging. Computer Methods in Biomechanics and Biomedical Engineering, 3:335-349, 2001.

7. S. Balac, H. Benoit-Cattin, T. Lamotte, and C. Odet. Analytic solution to boundary integral computation of susceptibility induced magnetic field inhomogeneities. Mathematical and Computer Modelling, 39:437455, 2004.

8. C.R. Camacho, D.B. Pleves, and R.M. Henkelman. Non-susceptibility artifacts due to metallic objects in MR imaging. JMRI, 5:75-88, 1995

9. J.A. Malko, J.C. Hoffman, and P.J. Jarret. Eddy-current induced artifacts caused by a MR compatible halo device. Radiology, 173:563-564, 1989.

10. L.H. Bennett, P.S. Wang, and M.J. Donahue. Artifacts in magnetic resonance imaging from metals. J. appl. phys., 79(8):4712-4714, 1996.

11. J.G. Sled and G.B. Pike. Standing-wave a RF penetration artifacts caused by elliptic geometry: an electrodynamic analysis of MRI. IEEE Trans. Med. Imag., 17(4):653-662, 1998.

12. M. Sadiku, editor. Numerical techniques in electromagnetics. CRC Press, 2000.

13. J. Jin. The finite element method in electromagnetics. John Wiley \& Sons, 1993.

14. F. Bloch. Nuclear induction. Physical Review, 70(7):460-474, 1946.

15. G. Sebastiani and P. Barone. Mathematical principles of basic magnetic resonance imaging in medicine. Signal Processing, 25:227-250, 1991

16. P.F. Hsieh and Y. Sibuya. Basic theory of ordinary differential equations. Springer, 1999.

17. W. Walter. Ordinary differential equations. Springer, 1998.

18. T.O. Levante, M. Baldus, B.H. Meier, and R.R. Ernst. Formalized quantum mechanical Floquet theory and its application to sample spinning in nuclear magnetic resonance. Molecular Physics, 86(5):1195 1212,1995

19. A.D. Bain and R.S. Dumont. Introduction to Floquet theory: The calculation of spinning sideband intensities in magic-angle spinning NMR. Concepts in Magnetic Resonance, 13(3):159-170, 2001.

20. F. Casas, J.A. Oteo, and J. Ros. Floquet theory: Exponential perturbative treatment. J. Phys. A, Math. Gen., 34(16):3379-3388, 2001.

21. W. L. Hart. The Cauchy-Lipschitz method for infinite systems of differential equations. American Journal of Mathematics, 43(4):226-231, 1921

22. S. Balac and M. Sadkane. On the computation of eigenvectors of a symmetric tridiagonal matrix: comparison of accuracy improvements of Givens and inverse iteration methods. Technical report, Laboratoire de Mathématiques Appliquées de Lyon, France, 2003. http://hal.archives-ouvertes.fr/hal00137149.

23. G. W. Stewart and J.-G. Sun. Matrix perturbation theory. Academic Press, New York, 1990.

24. J.D. Jackson. Classical electrodynamics. John Wiley and Sons, 1999.

25. H. Benoit-Cattin, G. Collewet, B. Belaroussi, H. Saint-Jalmes, and C. Odet. The SIMRI project : a versatile and interactive mri simulator. J. Magn. Reson. Imaging, 173:97-115, 2005.

26. R. A. Horn and C. R. Johnson. Matrix Analysis. Cambridge University Press, 1985.

27. J. A. Nyenhuis and O. P. Yee. Simulation of nuclear magnetic resonance spin echoes using the Bloch equation: Influence of magnetic field inhomogeneities. Journal of Applied Physics, 76(10):6909-6911, 1994.

28. D. E. Rourke. Solutions and linearization of the nonlinear dynamics of radiation damping. Concepts in Magnetic Resonance, 14(2):112-129, 2002

29. D. Zill. A first course in differential equations. ITP, 1997.

30. O.C. Zienkiewicz and R.L. Taylor. The finite element method. Mac Graw Hill, 1989.

31. S. Balac, G. Caloz, G. Cathelineau, B. Chauvel, and J.D. De Certaines. An integral representation method for numerical simulation of MRI artifacts induced by metallic implants. Journal of Magnetic Resonance in Medicine, 45:724-727, 2001 